\title{
Collective learning in news consumption
}

\author{
Niccolò Pescetelli ${ }^{1 *}$, Alex Rutherford ${ }^{1}$, Albert Kao $^{2}$, and lyad Rahwan ${ }^{1}$ \\ ${ }^{1}$ Max Planck Institute for Human Development, Berlin, 14195, Germany \\ ${ }^{2}$ Santa Fe Institute, Santa Fe, NM, 87501
}

\begin{abstract}
In a complex digital space-where information is shared with- 50 out vetting from central authorities and where emotional con- 51 tent, rather than factual veracity, better predicts content ${ }_{52}$ spread-individuals often need to learn through experience which news sources to trust and rely on. Although public and experts' intuition alike call for stronger scrutiny of public information providers, and reliance on global trusted outlets, there is a statistical argument to be made that counter these prescrip- ${ }^{56}$ tions. We consider the scenario in which news statements are ${ }^{57}$ used by individuals to achieve a collective payoff-as is the case 58 in many electoral contexts. In this case, a plurality of indepen- 59 dent though less accurate news providers might be better for 60 the public good than having fewer highly accurate ones. In a 61 carefully controlled experiment, we asked people to make binary forecasts and rewarded them for their individual or collective performance. In accordance with theoretical expectations, we found that when collectively rewarded people learned to rely more on local information sources and that this strategy accrued better collective performance. Importantly, these effects positively scaled with group size so that larger groups benefited more from trusting local news sources. We validate these claims ${ }^{68}$ against a real-world news dataset. These findings show the im- 69 portance of independent (instead of simply accurate) voices in 70 any information landscape, but particularly when large groups 71 of people want to maximize their collective payoff. These results suggest-at least statistically speaking - that emphasizing collective payoffs in large networks of news end-users might foster resilience to collective information failures.
\end{abstract}

news consumption; collective learning; judgment aggregation; group size; 7 forecasting

Correspondence: pescetelli@mpib-berlin.mpg.de

\section{Introduction}

In the run up to the 2020 US election, the collective patterns 82 of news consumption will be again under scrutiny. There is 83 a great deal of attention on trying to understand a complex 84 digital environment where information is transmitted with- 85 out vetting from central authorities and where the emotional 86 content of news, rather than their factual veracity, better pre- 87 dicts news spread $(1,2)$. In such environments, the reliability 88 of information sources must often be learned through experi- 89 ence rather than being established $a$ priori, with far-reaching 90 implications for society. Previous research has primarily fo- 91 cused on the dynamics by which rumors, from conspiracy 92 theories to malicious fake news, spread in a network of indi- 93 viduals (3-8). In response to the decrease in trust to a rapidly 94 shifting news landscape, the normal reaction of policy mak- 95 ers and public observers alike has been, understandably, to 96 stress the importance of well-known and trusted sources, in- 97 stead of relying on smaller information sources, social media 98 and private messaging channels $(9,10)$.

Centralizing information among fewer accurate global sources seems a sensible and intuitive strategy for increasing accuracy of people's knowledge and making it easier to maintain trust and reputation among content providers. However, recent theoretical and empirical evidence from psychology and animal behavior seems to suggest that, under specific circumstances, a group of individuals exclusively following global information sources might pay an accuracy cost when opinions are aggregated across the population, compared to individuals exclusively following local (but loosely accurate) independent information sources $(11,12)$. We call the former a consumer strategy and the latter a sleuth strategy. This collective accuracy cost is paid because of the correlations in information arising among individuals when they adopt a consumer strategy (13-17). Notice that here we do not focus on fake news producers (i.e., content that is intentionally deceptive). Rather, our point is that smaller (but still truth-seeking) local information sources are highly valuable for large groups of individuals who want to maximize their collective rather than individual accuracy.

Importantly, the question of which information source to trust (whether global or local) strongly depends on whether individuals are trying to solve an individual or collective optimisation problem. Individuals maximizing private payoffse.g., investors making private financial decisions - are better off by following an accurate global source (consumer strategy) as this will, over time, accrue a greater number of accurate predictions. However, when a large group of individuals tries to maximize a collective payoff - as in the case of voting on economic policies-following less accurate but independent sources (sleuth strategy) will maximize collective performance (12). This is clearly seen in Figure 1a. Assuming each individual can listen to only one news source, individuals following a consumer strategy (left) will be individually more accurate over time (sum over rows) than if following a sleuth strategy (right): $30 \% \mathrm{vs}$. $40 \%$ error rate respectively in the figure's example. However, when opinions are aggregated, e.g., via majority rule, collective decisions will benefit from individuals adopting a sleuth strategy (30\% vs. $20 \%$ error rate), because the risk of error can now be distributed among individuals. This effect is closely related to the Condorcet jury theorem, whose statistical properties have been thoroughly described in the literature $(13,16,17)$. Like in other Condorcet situations, the benefits of aggregating loosely predictive independent cues scales up with group size. In our news scenario, we expect that the benefit of individuals adopting a sleuth strategy (compared to a consumer strategy) on collective accuracy will increase over group size. 

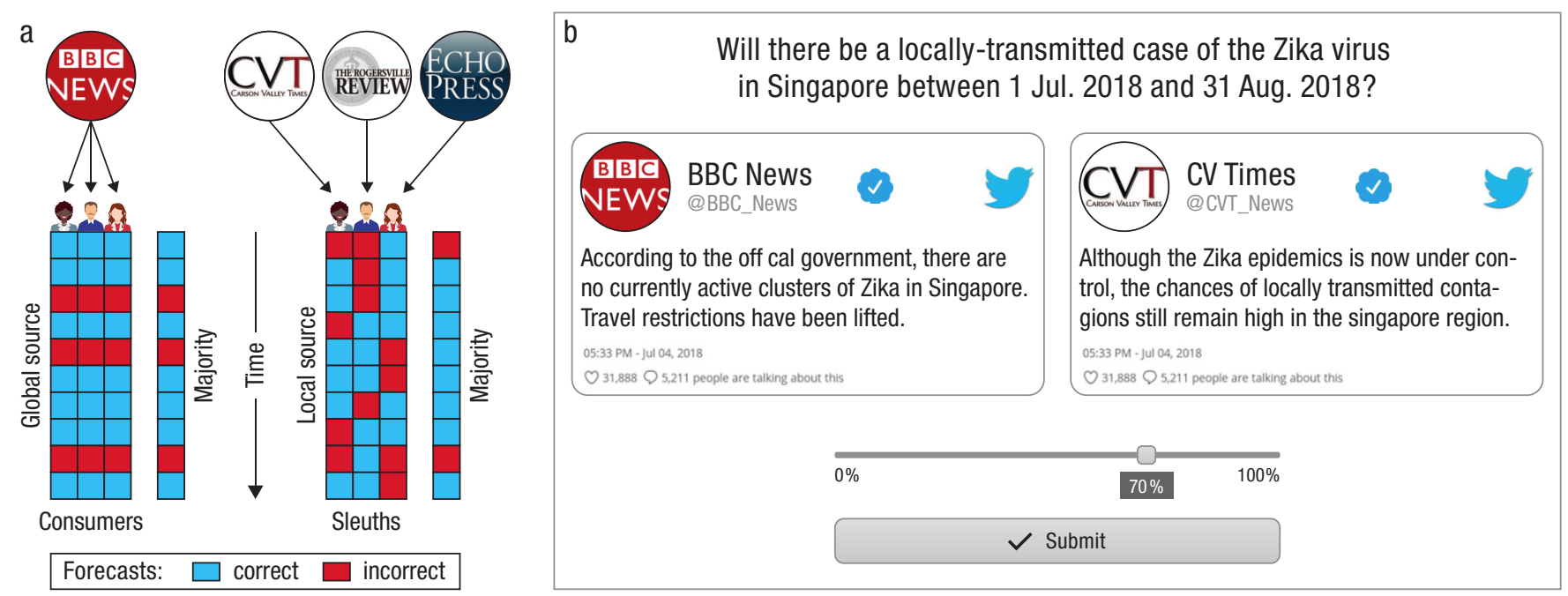

Fig. 1. a) Individuals select one news source to gather more information to make a binary decision (e.g., a forecast about the future). The 'global' news source on the left (e.g., BBC News) is more accurate, on average over time, than 'local' news sources on the right (e.g., local newspapers). However, because of its higher visibility, it generates high correlations among individuals in the population, aligning opinions and thus errors. When votes are aggregated on each round across individuals (e.g., via majority rule), the aggregate of local information sources can generate higher collective payoffs (although not necessarily higher individual payoffs). b) Representation of the experiment's interface. Participants are exposed to a sequence of forecasting problems surrounding realistic events (e.g., 'Will there be a case of Zika virus before August 2018?'). They can use relevant information provided by two news agencies in the form of tweets, either supporting the possibility of the event (e.g., tweet on the right) or opposing it (e.g., tweet on the left). Notice that 'BBC News' is used only for illustrative purpose, as an example of high-visibility global news source, and was not used in the actual experiment.

In a carefully controlled experiment, we study the mecha- 133 nisms underlying individual and collective news consump- 134 tion in binary forecasting tasks. We investigate the effect of ${ }_{135}$ payoff (individual vs. collective) and group size on (a) in- 136 dividual and collective accuracy, and (b) on people's prefer- ${ }^{137}$ ences for a global news source or a local one, as they expe- 138 rience over time positive $v s$. negative outcomes. Individu- 139 als acting in groups of varying sizes and under individual or 140 collective incentives used information provided by two alter- 141 native news agencies in the form of tweets to solve fictional ${ }_{142}$ but realistic prediction problems. Problems were independently selected by the IARPA Hybrid Forecasting challenge and their true outcomes were unknown at the time the experiment was run. Crucially, the accurate news agency (the 144 global cue) produced correlated errors across group mem- 145 bers, while the other (local cue) did not. To isolate the effect 146 of individual and collective learning we manipulate only the 147 information profile of local and global news sources, while 148 keeping all other contextual variables (like prestige and pop- 149 ularity of the news source) constant. For this reason, news 150 agencies in the experiment were randomly selected, for each 151 participant, from a pool of real but not well-known newspa- 152 pers (known by less than 5\% of our participant's pool). In ${ }^{153}$ other words, 'BBC News' is used in the figure only for illus- 154 trative purposes as a real-world example of a 'global' news 155 source, but was not used in the actual experiment. News 156 agencies that appeared in the experiment were assigned the 157 role of global or local cue, and attributes like Twitter follow- 158 ers and likes were matched across agencies and/or random- 159 ized across rounds.

We show that when collectively rewarded-but not when in- 162 dividually rewarded - groups of human volunteers learn to ${ }_{163}$ favor local independent news sources over more reliable but ${ }_{164}$ global ones, and that this has a significant impact on long- 165 term collective performance. This effect is consistent with theoretical predictions of a collective reinforcement learning model and scales up by group size. We test our claims against a large real-world news dataset and find no evidence that these effects may not operate also in real life. We discuss how these results are expected to play out in real online environments that feature millions of individuals sharing overlapping information sources (such as on social media), and how they can inform policies surrounding online news consumption and the design of algorithms to prevent misinformation.

\section{Results}

Collective rewards favor large groups Our hypothesis was that collective incentives benefit collective accuracy in large groups because, over time, people learn to rely more on private sources of information than if they received individual payoffs (12). In agreement with this hypothesis, we found collectively rewarded groups to achieve higher accuracy rates over time, compared to groups whose members are individually rewarded (Figure 2a, Tables 1 and S2). Due to the covariation between group size $(N)$ and noncompliance (see Supplementary Information $\S 1 \mathrm{D}$ and Table S1), we recover the causal effect of group size by local average treatment estimate $\left(N^{*}\right)$, capturing the quasiexperimental variation of our randomization process (18). Table 1 shows a binomial regression model on group accuracy as dependent variable, with fixed effects for group size and incentive (baseline:individual), which performed better (AIC compared) than alternative models. Results showed a negative main effect of group size $(\beta=-0.097, S E=$ $0.025, z=-3.87, p<0.001)$ suggesting a disadvantage of large individually rewarded groups over smaller ones. An interaction between group size and incentive was observed ( $\beta=.40, S E=.18, z=2.16, p=.03$ ), suggesting an advan- 
a

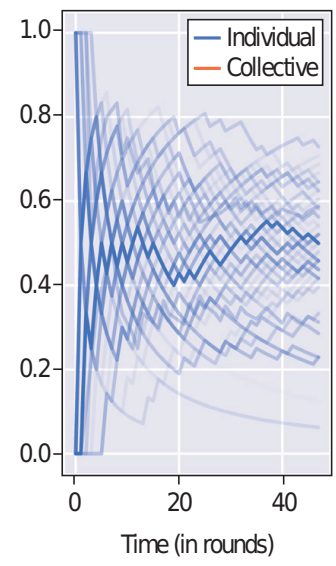

C

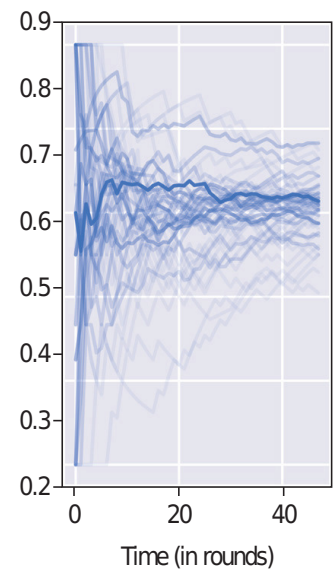

Cumulative group accuracy

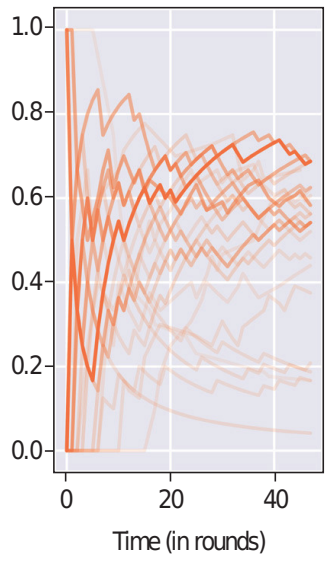

Time (in rounds) b

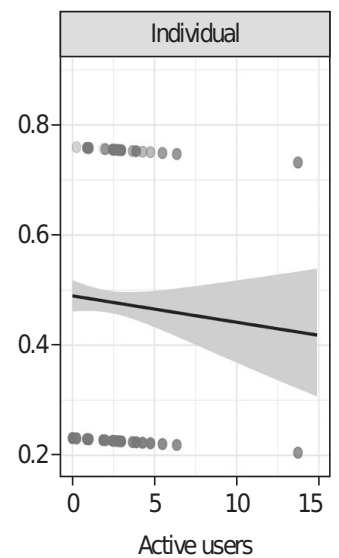

Group accuracy as a function of group size and incentive

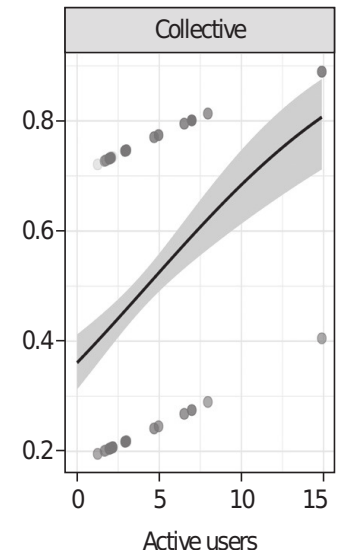

d Agreement with global cue as a function of cue agreement and incentive
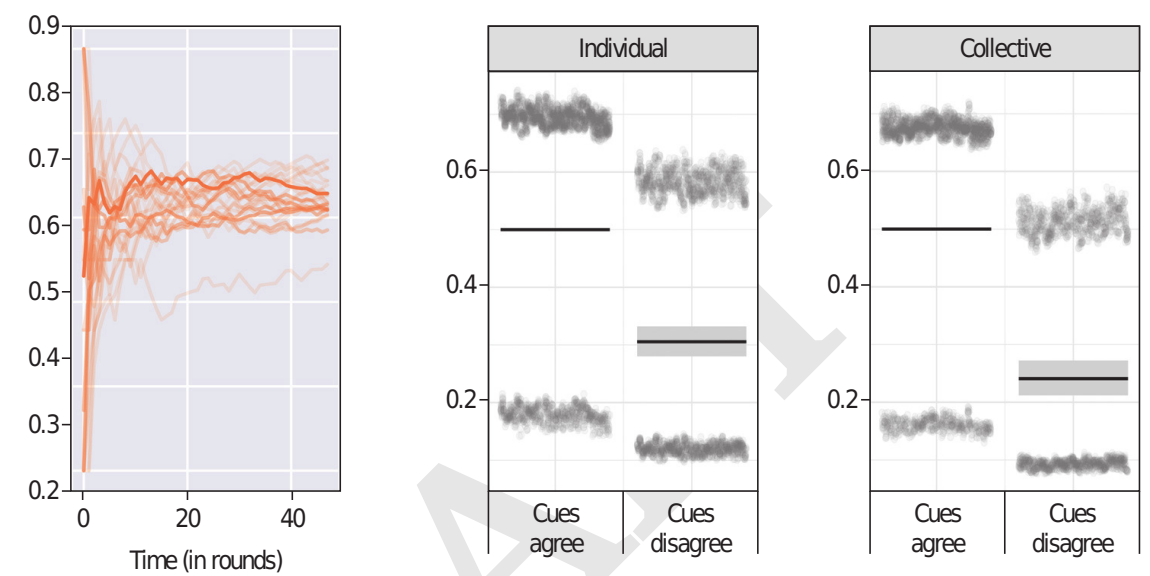

Fig. 2. (a) Cumulative group accuracy (y-axis) as a function of time (x-axis), group size as number of active users (saturation), and incentive (left-right panel). Large collectively rewarded groups tended to perform better over time than individually rewarded groups, surpassing the $70 \%$ limit of the global cue. (b) Partial residual plot of the effect of number of active users (x-axis) and incentive condition (left-right panel) on collective accuracy (y-axis). Solid lines represent model fit. Error bands represent confidence intervals (contrast to baseline value). (c) Cumulative agreement rate between individual responses and global cue information ( $y$-axis) as a function of time ( $x$-axis), number of active users (saturation), and incentive (left-right panel). (d) Partial residual plot of the effect of inter-cue conflict (x-axis) and incentive condition (left-right panel) on the individual cue preference (y-axis). Individual cue preference is computed as the agreement between individual choices and global cue. Solid lines represent model fit. Error bands represent confidence intervals (contrast to baseline value).

\begin{tabular}{lrrrl} 
Effects & Estimate & SE & z-value & p-value \\
\hline (Intercept) & 0.125498 & 0.067160 & 1.869 & 0.061673. \\
size & -0.097348 & 0.025145 & -3.871 & $\mathbf{0 . 0 0 0 1 0 8} * * *$ \\
incentive $_{\text {collective }}$ & -0.208918 & 0.143200 & -1.459 & 0.144586 \\
time & 0.073134 & 0.067327 & 1.086 & 0.277367 \\
size:incentive $_{\text {collective }}$ & 0.108593 & 0.038060 & 2.853 & $\mathbf{0 . 0 0 4 3 2 8} * *$ \\
size:time $_{\text {incentive }_{\text {collective: }} \text { :time }}$ & -0.036593 & 0.025315 & -1.446 & 0.148305 \\
size:incentive $_{\text {collective:time }}$ & 0.182450 & 0.143190 & 1.274 & 0.202600 \\
& -0.003347 & 0.038070 & -0.088 & 0.929934
\end{tabular}

Table 1. Generalized linear model of binomial family with logit link. Group accuracy as a function of group size $\left(N^{*}\right)$, incentive condition and time. Model: $g r o u p A c c u r a c y \sim$ $N^{*} *$ incentive *time.

tage for large groups to be rewarded collectively instead of 173 individually. Similarly, the group's average number of ac- 174 tive users strongly predicted group accuracy in the collec- 175 tive incentive condition $(\beta=0.15362, S E=0.03030, z=$ $5.070, p<001)$ but not in the individual incentive condi- ${ }^{176}$ tion $(\beta=0.019, S E=0.019, z=-1.007, p>0.3)\left(\right.$ Figure $^{177}$ $2 b)$. Furthermore, models including time as a fixed effect ${ }_{179}^{178}$ showed a trend for increased accuracy in collectively rewarded groups over time, indicating an effect of learning (Table S3).

The same models were fitted on individual rather than group accuracy, to test whether the observed improvement in group accuracy was a product of improved individual accuracy. To account for the non-independence of individual level data, we 
ran mixed-effects models. The model included group size, 237 incentive and time as fixed effects and time as random effect ${ }_{238}$ (Table S4). Individual accuracy of individuals in collectively $2 з 9$ rewarded groups was not superior over accuracy of individ- 240 uals in isolation $(\beta=0.07, S E=0.04, z=1.620, p>0.1)_{241}$ nor the effect interacted with group size $\left(\beta=-0.015, S E={ }_{242}\right.$ $0.043, z=-0.356, p>.1)$. The results were robust across ${ }_{243}$ a wide variety of alternative models, including adding other ${ }_{244}$ fixed effects (e.g., cue agreement), and under alternative ${ }_{245}$ grouping variables such as participant id, group id or no 246 grouping at all (simple GLM). Overall the findings suggest ${ }_{247}$ that group accuracy improved, notwithstanding individual ac- 248 curacy remaining unaffected by our incentive and group size ${ }_{249}$ manipulation. Collective accuracy improvement emerged in- 250 stead at the aggregate level, when a majority rule was applied ${ }_{25}$ to independent - rather than correlated-individual judge- 252 ments. Similarly to other "wisdom of crowds" effects, we ${ }_{253}$ showed that collective accuracy can improve (here via incentive manipulation) even though individual accuracy within the crowd remain unaltered.

Collectively rewarded individuals prefer local cues. To understand the mechanisms underlying the observed advantage of large collectively rewarded groups, we analyzed individual level decisions. Our hypothesis was that collectively rewarded people learn to rely more on private information than when individually rewarded (12). We found that individual preferences for the global cue were affected by group size and incentive (Figure 2c). Given that trial-level preferences for the global $v s$. local cues were not directly observable, we inferred them from the observed participant behavior. We operationalize individual preferences for the global cue as the agreement between the binarized participant's choicenamely, which event the participant believed was more likely to occur-and the global cue's binarized evidence-namely, whether the global cue's tweet suggested the event was likely to occur or not. This leverages on the intuition that if participants deemed the global cue accurate, they should use, and their forecasts should match, the information provided by it. To account for non-independence of individuals belonging to same groups, a generalized linear mixed-effects model on the response-global agreement measure as depen- 254 dent variable showed that individuals in isolation favored ${ }_{255}$ global cues ( $\beta=0.893, S E=0.052, z=17.01, p<0.001)$, ${ }_{256}$ as expected by the higher accuracy of this cue over the local ${ }_{257}$ one (Table 2). When cues were in agreement, collectively re- 258 warded participants in smaller groups tended on average to 259 use global cues more than individually rewarded ones $(\beta=260$ $0.258, S E=0.077, z=3.353, p<.001$ ), perhaps due to the ${ }_{26}$ fact that they experienced a noisier mapping between choices 262 and outcome, than individually rewarded players. Impor- 263 tantly however, when cues were in disagreement-and thus 264 participants had to decide which one to listen to-collectively 265 rewarded participants preferred the local cue over the global 266 one more often than individually rewarded participants $\left(\beta={ }_{267}\right.$ $-0.366, S E=0.093, z=-3.923, p<.001$ ) (Figure 2d). We 268 expected this latter effect to scale with group size, given that ${ }_{269}$ larger collectively rewarded groups should benefit more from 270 adopting a local information strategy. However, the effect was significant only when excluding groups containing idle participants $(\beta=-0.68, S E=0.32, z=-2.09, p=.03)$, Table S5.

Finally, at the end of the experiment, we asked participants to explicitly report what they believed the accuracy of each news source was and which news source they mostly relied on in their decisions. A higher number of individually rewarded participants than collectively rewarded participants reported to have relied more heavily on the global cue. On the contrary, more collectively rewarded than individually rewarded participants reported to have relied more heavily on local cues (Figure 3a). However, when asked to express their intuitions about each cue's reliability as a percentage of correct answers, only a numerical difference was observed whereby participants in the collective condition tended to prefer local cues (Figure 3b).

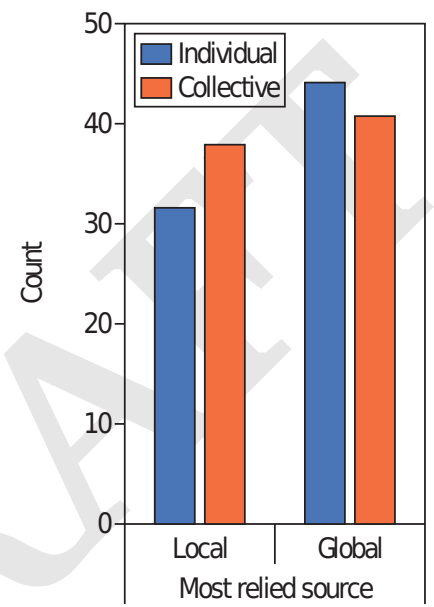

b Reported accuracy difference (global-local)

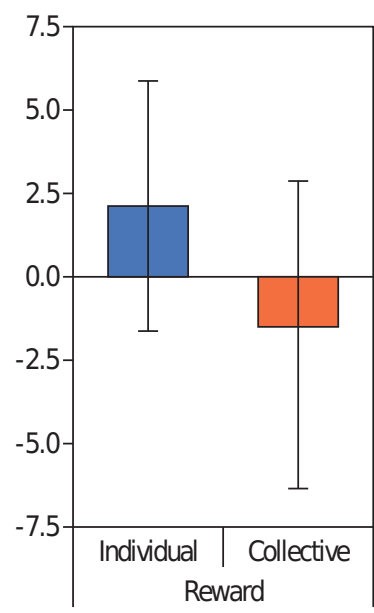

Fig. 3. Individual beliefs as explicitly reported in the debrief questionnaire presented to participants at the end of the experiment. a) Number of participants (y-axis) who reported a preference (i.e., to rely in their judgments more heavily on) for the local or global information source. b) Reported accuracy difference between local and global information source in the two payoff conditions. Groups with idle participants were removed. Error bars represent standard errors.

Decision confidence is not influenced by incentive and group size We then turned to ratings of subjective confidence in participants' decisions. Given that each binary decision was rated by participants on a continuous scale (range "The event will surely happen" to "The event will surely not happen"), we defined confidence as $50 \%+|f-50 \%|$, namely the distance of the forecast $f$ from the uncertainty boundary $50 \%$ plus the probability of getting the correct answer by random guessing. We fitted this measure to a mixed-effect model to measure the effect of our manipulation, time and cue agreement on this measure of confidence. Several models were compared with AIC. The winning model included fixed effects for size, incentive and inter-cue agreement, and random effects for participants, groups and time (Table 3$)$. Results show that confidence was negatively affected by inter-cue disagreement $(\beta=-0.171, S E=0.021, d f=$ $9483.042, t=-8.069, p<7.97 e-16)$. Furthermore, an in- 


\begin{tabular}{lrrrl} 
Effects & Estimate & SE & z-value & p-value \\
\hline (Intercept) & 0.89308 & 0.05248 & 17.019 & $<\mathbf{2 e - 1 6} * * *$ \\
size & 0.09602 & 0.05121 & 1.875 & 0.0608 . \\
incentivecollective & 0.25887 & 0.07721 & 3.353 & $\mathbf{0 . 0 0 0 8} * * *$ \\
cueAgreementFalse & -0.86576 & 0.06138 & -14.106 & $<\mathbf{2 e - 1 6} * * *$ \\
size:incentivecollective & -0.08177 & 0.07703 & -1.062 & 0.2884 \\
size:cueAgreementFalse & -0.07554 & 0.06243 & -1.210 & 0.2263 \\
incentivecollective:cueAgreementFalse & -0.36691 & 0.09354 & -3.923 & $\mathbf{8 . 7 6 e - 0 5} * * *$ \\
size:incentivecollective:cueAgreementFalse & 0.03351 & 0.09354 & 0.358 & 0.7202
\end{tabular}

Table 2. Preference for global cues as a function of cue agreement, group size $\left(N^{*}\right)$ and incentive structure. Model: agreementWithGlobal $\sim$ size $*$ incentive $*$ cueAgreement $+(1 \mid$ playerId : gameId $)+(1 \mid$ time $)$.

teraction between group size and cue agreement was found ${ }_{314}$ $\left(\beta=-0.060, S E=0.020, d f=9482.896, t=-2.957, p={ }_{315}\right.$ 0.003 ) suggesting that individuals in large groups tended to ${ }_{316}$ show less confidence in their forecasts when facing disagree- ${ }_{317}$ ing cues, compared to individuals in groups of smaller size. ${ }_{318}$ No interaction was found between size and incentive, sug- ${ }_{319}$ gesting that our accuracy findings were not entirely explained ${ }_{320}$ by changes in individuals' decision confidence.

\section{Newspapers content similarity correlates with degree of lo- ${ }^{323}$} cality To understand whether similar effects may be op- ${ }^{324}$ erating not just in the lab but also in real life, we con- ${ }^{325}$ ducted some preliminary observational analyses on pub- ${ }^{326}$ licly available news datasets. We used the News Aggrega- ${ }^{327}$ tor Dataset, freely available at Kaggle.com, from the UCI ${ }^{328}$ Machine Learning Repository (19). This dataset contains ${ }^{329}$ headlines, URLs, and categories for 422,937 news stories ${ }^{330}$ collected by a web aggregator between March 10th, $2014^{331}$ and August 10th, 2014, and 10986 unique publishers, rang- ${ }^{332}$ ing from popular ones (e.g., Reuters and The Guardian) to ${ }^{333}$ smaller ones. News categories in the dataset include busi- ${ }^{334}$ ness, science and technology, entertainment, and health. We ${ }^{335}$ chose this dataset over other available ones because different ${ }^{336}$ news articles that refer to the same news item (e.g., several ar- ${ }^{337}$ ticles about recently released employment statistics) are cat- ${ }^{338}$ egorized together under the same story ID. There are $7230^{339}$ unique stories covered in the dataset. This feature allowed us ${ }^{340}$ to ask whether larger publishers (global news sources) tend to ${ }^{341}$ be more correlated in terms of stories covered and their story ${ }^{342}$ content than smaller ones (local news sources). Given that ${ }^{343}$ information about the size or popularity of the publisher was ${ }^{344}$ not available in the dataset, we used the presence of a pub- ${ }^{345}$ lisher in the dataset itself (in number of entries) as a proxy ${ }^{346}$ measure, under the assumption that larger newspapers would ${ }^{347}$ be more prolific and thus appear more often in aggregator ${ }^{348}$ searches. This measure turned out to be a surprisingly good ${ }^{349}$ approximation of a publisher's size ('globality'). According ${ }^{350}$ to it, the top publisher was Reuters, followed by several well- ${ }^{351}$ known others like Bloomberg and the Huffington Post. At the bottom of the distribution (with just one entry in the dataset) were several smaller publishers (e.g., Yale Daily News or The ${ }^{352}$ Cameron Herald). In the future, more precise measures may 353 be obtained by looking at readership volume, popularity (e.g., 354 Twitter followers) or even geographic indicators of 'locality' 355 (e.g., where a newspaper can be purchased).

We found inverse correlations existing between the degree of locality of a publisher and both the similarity of their headlines for each story covered as well as the similarity in the set of stories covered (Figure 4). Given the large size of the dataset, we could not compute all pairwise comparisons. We thus took Reuters as our global reference and compared other publishers' measures with respect to it. A small but significant negative correlation existed between a publisher degree of locality-defined as the difference in output with respect to Reuters as described above-and the average similarity of the headlines, for each story covered by both publishers $(r h o=-.062, p<.001)$. In other words, the larger the publisher the more likely its story headlines were to Reuters' headlines covering the same story. Headline similarity was computed as pairwise cosine similarity of term frequency-inverse document frequency (tf-idf) vectors. Notice that, as expected, publishers with the highest degree of locality (rightmost datapoints in Figure 4a) also show a higher variability in their headline similarity with Reuters. Intuitively this makes sense, as smaller outlets may cover niche topics or serve smaller local regions than global newspapers. Overall, these findings suggest that smaller news sources tend to be more diverse (i.e., more independent) in their content than larger global news sources.

Furthermore, a strong negative correlation was found between the similarity in the stories covered-defined as the Jaccard index between the sets of stories covered - and the degree of locality of a publisher, as defined above ( $r h o=$ $-0.79, p<.001$ ) (Figure 4b). Together, these observations on real world data are consistent with our findings. They suggest that large publishers may indeed be more correlated with each other (both in terms of the subset of stories covered and their linguistic similarity when covering the same story) than smaller ones. Nevertheless, we warn caution when interpreting these preliminary findings, and invite researchers to use different methods and larger datasets to put to a test these claims.

\section{Discussion}

In this paper, we have explored patterns of collective learning in the presence of correlated information and payoffs among group members. In a forecasting task where indi- 


\begin{tabular}{lrrrrl} 
Effects & Estimate & SE & df & t-value & p-value \\
\hline Intercept) & 0.077 & 0.061 & 243.125 & 1.268 & 0.206 \\
size & 0.025 & 0.059 & 229.505 & 0.429 & 0.668 \\
incentive $_{\text {collective }}$ & 0.037 & 0.091 & 228.744 & 0.410 & 0.682 \\
cueAgreement $_{\text {False }}$ & -0.171 & 0.021 & 9483.042 & -8.069 & $\mathbf{7 . 9 7 e - 1 6} * * *$ \\
size:incentive $_{\text {collective }}$ & -0.113 & 0.089 & 226.638 & -1.274 & 0.203 \\
size:cueAgreement $_{\text {False }}$ & -0.060 & 0.020 & 9482.896 & -2.957 & $\mathbf{0 . 0 0 3} * *$ \\
incentive $_{\text {collective }}:$ cueAgreement $_{\text {False }}$ & -0.050 & 0.031 & 9491.962 & -1.588 & 0.112 \\
size:incentive $_{\text {collective }}:$ cueAgreement $_{\text {False }}$ & 0.022 & 0.030 & 9490.204 & 0.729 & 0.465
\end{tabular}

Table 3. Individual confidence as a function of inter cue agreement, size and incentive structure. Model: confidence $\sim$ size $*$ incentive $*$ cueAgreement + $(1 \mid$ playerId: gameId $)+(1 \mid$ time $)$.

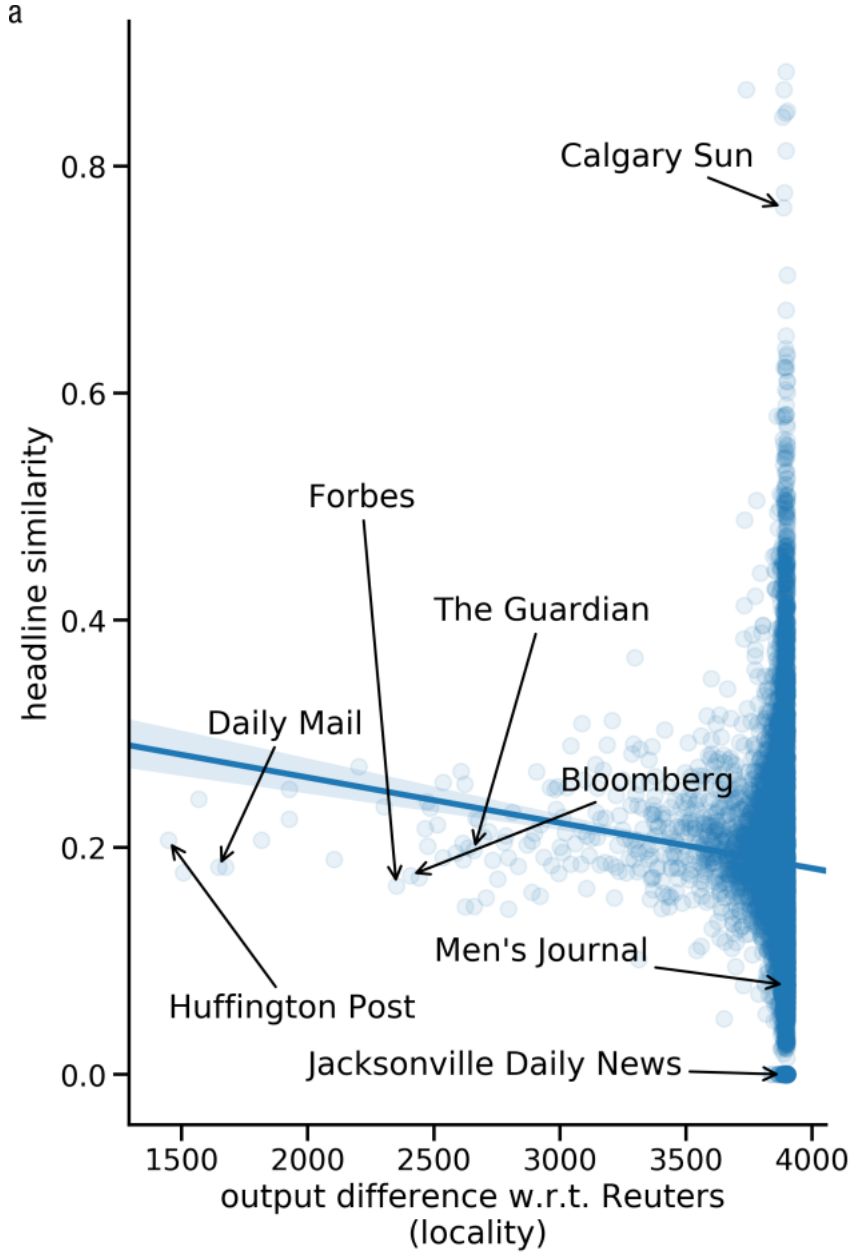

b

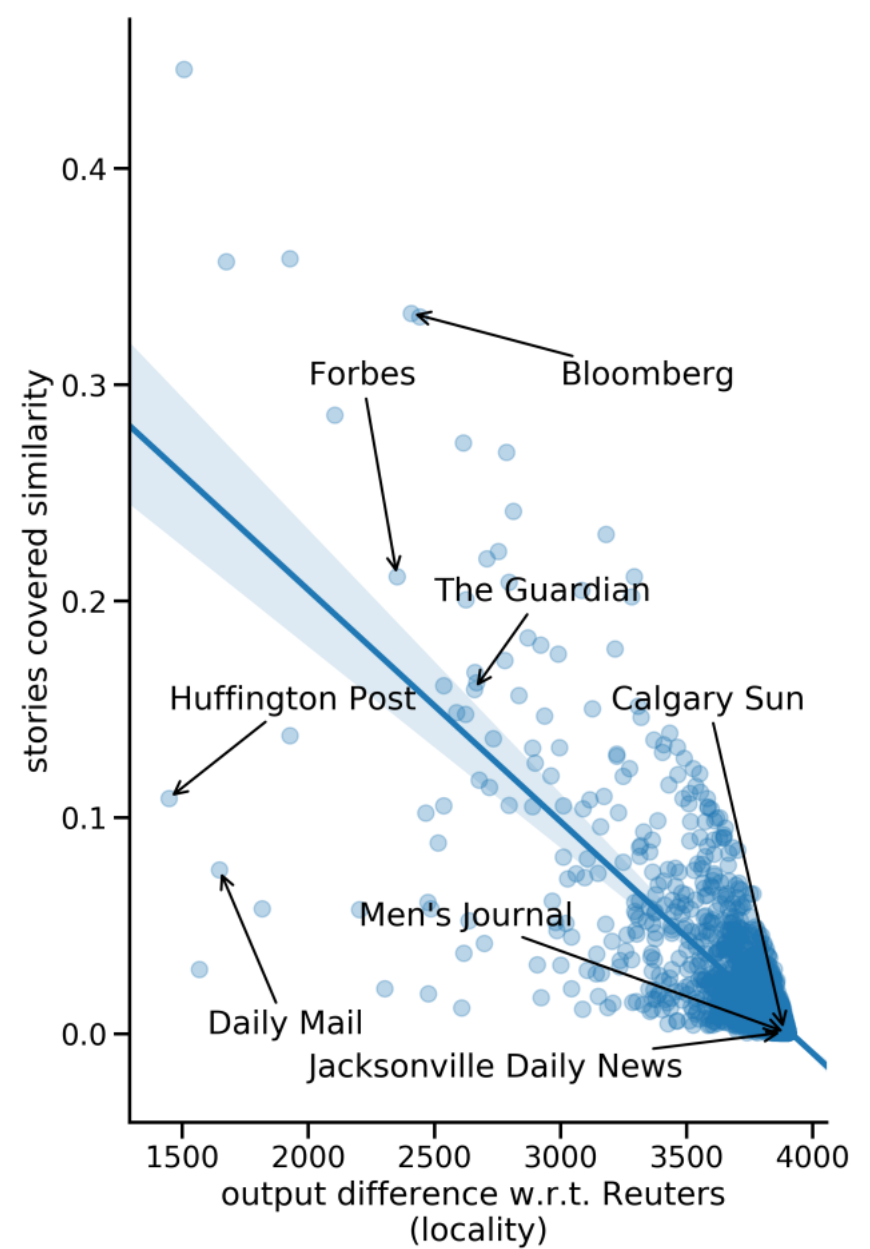

Fig. 4. (a) Negative correlation existing between publishers' headline similarity and their output difference with respect to Reuters (i.e., a proxy measure of 'locality'). Headline similarity was calculated as average pairwise similarity between pairs of newspapers headlines covering the same story, using the term frequency-inverse document frequency (tf-idf) method. Locality was calculated using as a proxy the difference in output between Reuters (the most represented and prolific publisher in the data set) and other publishers. This measure is a rough approximation of the locality of a publisher. Publishers that are more represented in the dataset (on the left-hand side of the panel) also tend to be larger and well-known publishers, compared to publishers on the right. (b) Negative correlation existing between similarity in the set of stories covered by Reuters and other publishers, and their locality (i.e., as proxied by output difference with respect to Reuters). Stories similarity was computed as the Jaccard similarity of the set of stories covered by each publisher with respect to the set of stories covered by Reuters. The dataset is publicly available at Kaggle.com (19)

viduals need to use information conveyed by two alternative ${ }_{363}$ news sources to predict realistic future events, we showed ${ }_{364}$ that differences in news information consumption emerge de- 365 pending on group size and the payoffs that individuals are 366 trying to maximize. When trying to maximize their indi- 367 vidual payoff, people learned to rely on the most accurate 368 news source, as expected from reinforcement learning prin- 369 ciples $(20,21)$. Although beneficial for individual performance, this 'consumer' strategy hindered aggregate performance in our task because it made groups sensitive to collective shocks: When the global news source was wrong, every consumer was wrong at once. Accordingly, when trying to maximize a collective payoff-here, the accuracy of the majority-people learned to optimally trade-off information 
accuracy with information independence, the latter being the ${ }_{427}$ extent to which information was shared across other members 428 of the group. We showed that this 'sleuth' strategy benefits 429 group (but not individual) performance, while leaving indi- 430 viduals' decision confidence largely unchanged. Finally, we ${ }_{431}$ test our claims against a real world news dataset and found no 432 evidence against them. Content provided by news publishers 433 was shown to be more similar (both in terms of stories cov- 434 ered and text headlines within each story) if it was from large 435 publishers than if it was from smaller ones.

How to deliver fair news reporting in the digital age is one ${ }^{437}$ of the challenges of our digital society. Worries about infor- ${ }^{438}$ mation quality have benefited 'reputable' news sources, with ${ }^{439}$ $26 \%$ people world-wide ( $40 \%$ in the US) increasingly rely- ${ }^{440}$ ing on trusted newspapers and a further $24 \%$ no longer re- ${ }^{441}$ lying on dubious sources altogether. Similarly, pivoting to ${ }^{442}$ paid subscriptions to counter lost public trust seems to fos- ${ }^{443}$ ter a 'winner-take-all' dynamic where people are willing to ${ }^{444}$ pay for only one newspaper, or alternatively to consume free ${ }^{445}$ algorithmic-curated news $(9,10)$. These documented trends ${ }^{446}$ seem to be at odds with consolidated findings in collective ${ }^{447}$ intelligence, namely the importance of having a plurality of ${ }^{448}$ independent voices for collective accuracy $(13,16,17)$. In ${ }^{449}$ large groups, aggregating information over loosely informa- ${ }^{450}$ tive but independent sources dominates over source accuracy ${ }^{451}$ $(12,17)$. Notice that according to this argument sources need still to be 'loosely informative' (i.e., above chance), so sources that intentionally spread 'fake news' or malicious content are not to be preferred regardless of the situation. Importantly, we are not suggesting to discard trusted sources of information, just to draw attention to the trade-off existing between accuracy and independence in matters of collective relevance. First, whenever people are motivated by individual interests (like making good judgements for one's own private good) people should rely on trusted accurate sources. Considerations about information independence are important only when people value being collectively, rather than individually, accurate. This might be the case when the news is used to inform society-wide debates over policies of collective relevance, in voting and during referenda. Our analysis of real world news suggests that these trends may not be just the product of a careful experimental design but may be at work in real life. Our observations suggest that indeed small publishers tend to be more diverse in the set of stories ${ }^{469}$ covered and in the content provided when covering the same story (as judged by our correlational analysis of newspaper headlines). Notice that no measure of news source accuracy was here provided, but only evidence that some degree of correlation exists among large popular publishers. Nevertheless, Ladha's work suggests that overall group accuracy can be independently raised both by raising mean accuracy of individual sources and by lowering their mean dependence ${ }_{477}$ (17). Considerations about news sources independence are ${ }_{479}^{478}$ thus important beyond what can be said about their accuracy ${ }^{479}$ and reliability.

Second, even when trying to maximize a collective payoff, ${ }_{482}$ mixed strategies theoretically dominate pure strategies alike ${ }_{483}$
(Figure 1C in (12)). To support this fact, it is likely that in our experiment people adopted a mixed strategy, alternating among different news agencies from trial to trial, as if using probability matching. In probability matching, people alternate between options (e.g., in multi-armed bandit tasks) proportionally to each option's expected reward instead of exploiting the most rewarding option. This allows them to balance exploration and exploitation $(22,23)$. Adopting a mixed strategy in a population can be adaptive in distributing risk among group members (24). Participants in our experiment showed some evidence of probability matching (see Supplementary material Extended Results), alternating among news options even in later stages of the experiment (Figure S4). Our claim thus, is not that collective accuracy is maximized in this task by a pure sleuth strategy, nor that participants exclusively used local news sources when collectively rewarded. Our evidence simply suggests that, in our binary forecasting task, pure sleuths dominate pure consumers when maximizing collective performance, and that people learned to rely more heavily on private information, when maximizing a collective rather than individual payoff. Furthermore, participants did not seem to follow simpler heuristics such as conformist beauty-contest strategies (see Supplementary material Extended Results), further confirming the use of an adaptive strategy when trying to predict trial outcomes.

Our findings add to existing empirical evidence that individual incentives can be suboptimal when trying to reap a collective rather than individual payoff (11). The study by Bazazi and colleagues shows that in the presence of uncertainty and social interaction, rewarding individuals for their individual performance increases people's propensity for herdingnamely copying others' solutions or majoritarian decisionsbecause it can alleviate the costs of individual learning (2528). Collective incentives can overcome this limitation. Our study further shows that not only social interaction, but much simpler choice-reward associations are enough to impact collective accuracy when one's payoff is coupled to other members in a group. Notice that in our study, individuals in a group never 'interacted' with one another, but simply experienced the same rewards as others in their group. By changing their incentive structure (individual $v s$. collective), people can be nudged to favor different information sources (global vs. local), which in turn determines the boundaries of their individual and collective performance. It is an open question whether in real-world settings providing collective incentives is sufficient to increase people's reliance on local independent news sources, and whether this would be sufficient to strengthen a network's resilience to misinformation. Collective decisions were defined in this study using majority, but similar mechanisms underlie many other aggregation rules (12). Notice that participants in our study were unaware of the distinction between local and global cues nor of the size of the group they were in, as confirmed by analyzing post-experiment survey data. From an individual point of view, participants only experienced a sequence of actionreward pairings and any other form of communication with other members of the group was not allowed. 
Our findings are consistent with some practices adopted in fi- 540 nance and economics. Investors maximising returns are often ${ }_{541}$ required to seek information exclusive to them and to avoid ${ }_{542}$ relying only on shared information (e.g., news articles, quar- 543 terly earnings statements) to more accurately price assets. 544 Diversification of portfolios is a common strategy to avoid 545 correlated crashes. They also point out some limitations of 546 widespread practices, like awarding bonuses to high perform- 547 ing individuals, which may incentivise consumer-like be- 548 haviors that are detrimental for companies. In the context 549 of online news consumption, the effect of decentralized lo- 550 cal preferences on society's resilience to misinformation is 551 still poorly understood, and current practices are exposing 552 the weak spots of the shift to digital. In our study, global ${ }_{553}$ and local cues were easily defined, but a clear distinction ${ }_{554}$ remains difficult in the real digital news ecosystem. Here, 555 news agencies themselves are likely to rely on third party in- 55 formation sources, like AP and Reuters, within a complex ${ }_{557}$ web of interdependencies that is difficult or impossible to un- 558 tangle. Although the news ecosystem is more complex than ${ }_{559}$ our two-agency toy world, we could not find any evidence 560 against our claims when looking at real-world news in our ${ }_{561}$ observational analysis. Thus, the collective learning mech- 562 anisms discussed in our work might be subtly shaping patterns of news consumption in the real world. Whether a so- ${ }^{563}$ ciety values individual or collective accuracy is difficult to ${ }^{564}$ measure, but our results suggest that people can be nudged ${ }^{565}$ toward news consumption patterns that favor individual or ${ }^{566}$ collective good. Far from making a policy recommendation, ${ }^{56}$ we simply call for greater attention to the cognitive processes ${ }^{568}$ underlying collective rather than individuals modes of news ${ }^{569}$ consumption. Our insights are supported only by a statisti- ${ }^{570}$ cal argument, a single (rather small-scale) experiment and an ${ }^{571}$ observational analysis of real-world news data. We thus rec- 572 ommend caution in interpreting or applying our results. Nev- ${ }^{573}$ ertheless, both our empirical and theoretical evidence stress ${ }^{574}$ the importance of maintaining - in the face of recent trends in ${ }^{575}$ digital news consumption patterns - a rich landscape of local ${ }^{576}$ independent news sources for our collective good.

\section{Methods}

Procedure The study was approved by MIT IRB. The full 581 procedure is reported in $\$ 1 \mathrm{C}$. Participants $(\mathrm{N}=232)$ were re- 582 cruited on Amazon Mechanical Turk and paid proportionally 583 to their time and performance in the game. They gave in- 584 formed consent before starting the game. The game was im- 585 plemented using the Empirica platform for group experimen- 586 tation, developed in the MIT Media Lab and publicly avail- 587 able at empirica.1y (29). Participants were randomized ${ }_{588}$ into two different incentive conditions (Incentive: collective ${ }_{589}$ vs. individual reward) and four different group sizes (Group 590 Size: 1, 3, 7 or 15). Group performance was calculated trial- 591 by-trial based on the majority decision. Participants waited 592 in an online waiting room to allow everyone to $\log$ in and ${ }_{593}$ read the instructions. If the room was not full by the end ${ }_{594}$ of 15 minutes, the game started with the number of partici- 595 pants available. The game consisted of a number consecutive 596 rounds in which participants had to answer realistic binary questions regarding geo-political events (see full problem and tweets list in Supplementary material), independently generated by the IARPA Hybrid Forecasting Competition-a national forecasting competition aiming at improving prediction accuracy of global events of geo-political relevanceand whose outcomes were unknown at the time of the experiment (e.g., "Before 8 September 2018, will Chinese tariffs on U.S. soybeans take effect?"). Within the present experiment, participants were told that the game would have simulated the outcome of each event and their performance would have been calculated based on their prediction within the game and the simulated outcome. They were thus explicitly incentivised to avoid using any private knowledge on the topic and use only the information available via the tweets that were provided in the experiment. Importantly, the use of private knowledge could have not improved their accuracy in the game as events in the game could be predicted only by the news sources made available to them. At the end of each round, participants received feedback on the response they individually provided, the response of the group's majority, and the simulated outcome (The event happened vs. The event did not happen).

Local and global information sources A detailed account of the generative model producing the stimuli is described in Supplementary material $\$ 1 \mathrm{~A}-\mathrm{B}$. Each participants was randomly paired with two news agencies selected from a pool of real but not well-known (as judged by the number of followers on Twitter) news magazines and newspapers. Less than $5 \%$ of participants reported to know the news agencies in advance. News sources provided on each round questionspecific information that either supported or went against a given question's outcome, thus making the question's event either more or less likely. All information was created ad hoc by the experimenter and was presented to participants as a short tweet from the newspaper's mock-up twitter account. Participants were debriefed about the fictional nature of this exercise. For the question "Before 8 September 2018, will Chinese tariffs on U.S. soybeans take effect?", an example of supporting tweet was "Latest reports suggest China will not back down from trade war and tariffs on US soybeans are expected to increase". An example of a tweet reducing the likelihood of the event was "US-China trade war: After months of escalation, both parties are ready to start finding a solution that will benefit both". On each round, a simulated outcome (e.g., "Yes, Chinese tariffs on U.S. soybeans have taken effect") was produced by the game.

In the back-end, one of the two news sources was chosen as global cue, while the other as local cue. Local and global news sources differed only (a) in their error rates-namely the rate of (mis)match between their tweet's information (Supporting vs. Contrary to the event) and the simulated round outcome (The event happens vs. The event does not happen - - set to $35 \%$ and $30 \%$ for the local and global cue respectively, and (b) in the correlation of their errors among group members (Figure 1a). In other words, on rounds when the global cue was accurate (inaccurate)-i.e., the tweet in- 
formation matched (mismatched) the simulated outcome-it 655 was accurate (inaccurate) for every member of the group. On ${ }_{657}^{656}$ the contrary, errors made by a participant's local cue were ${ }_{658}$ independent from errors made by other group members' 10- ${ }^{659}$ cal cues. Other news agency features were either randomized 661 (like logo, news agency name and side (left/right) on which ${ }_{663}^{662}$ the news agency appears) or kept constant (e.g., number of ${ }_{664}^{663}$ followers displayed on the tweet) across participants. A sur- ${ }_{666}^{665}$ vey asked participants at the end of the experiment their be- ${ }_{667}$ liefs about their group size, the cue they relied the most on, ${ }_{669}^{668}$ and each cue perceived accuracy rate.

Newspaper similarity Our analysis of real-world news data ${ }^{672}$ relied on three measures extrapolated by a publicly available ${ }^{673}$ news dataset, namely locality, headline similarity and simi- ${ }^{-675}$ larity in the set of stories covered. Locality was defined as 677 the absolute difference between number of occurrences of $a_{679}^{678}$ publisher in the dataset and the number of occurrences of the 680 publisher with the highest number of occurrences, which was ${ }^{681}$ Reuters in the dataset considered. Larger differences corre- 683 spond to smaller (more local) publishers. To calculate head- ${ }^{684}$ line similarity we first converted the raw headlines to a ma- 686 trix of TF-IDF features using the scikitlearn package. Then ${ }_{688}^{687}$ we calculated pairwise similarity between two headlines as 689 the dot product of their TF-IDF vectors. Finally, the simi- ${ }_{691}^{690}$ larity in the stories covered by two publishers was calculated 692 as the Jaccard similarity between the sets of stories covered ${ }_{694}^{693}$ by each publisher, namely the number of stories covered by 695 both newspapers over the total number of stories covered by ${ }_{697}^{696}$ either.

Replicability Data and analysis are made available online 70 via OSF (https://osf.io/x5hye).

Acknowledgements This research is based upon work sup- $-{ }_{705}^{704}$ ported in part by the Office of the Director of National In- 706 telligence (ODNI), Intelligence Advanced Research Projects ${ }_{708}^{707}$ Activity (IARPA), via contract number 2017-17061500006. 709 The views and conclusions contained herein are those of the ${ }_{711}^{710}$ authors and should not be interpreted as necessarily repre- 712 senting the official policies, either expressed or implied, of ${ }_{714}^{713}$ ODNI, IARPA, or the U.S. Government. The U.S. Govern- 715 ment is authorized to reproduce and distribute reprints for ${ }_{717}^{716}$ governmental purposes notwithstanding any copyright anno- 718 tation therein.

The authors kindly thank Dr. Nick Obradovich and Dr. Ab- ${ }^{720}$ dullah Almaatouq for their support and advice.
5. Alessandro Bessi. Personality traits and echo chambers on facebook. Computers in Human Behavior, 65:319-324, 12 2016. ISSN 07475632 doi: 10.1016/j.chb.2016.08.016.

6. Michela Del Vicario, Gianna Vivaldo, Alessandro Bessi, Fabiana Zollo, Antonio Scala, Guido Caldarelli, and Walter Quattrociocchi. Echo Chambers: Emotional Contagion and Group Polarization on Facebook. Scientific Reports, 6(1):37825, 12 2016. ISSN 2045-2322. doi: 10.1038/srep37825.

7. A. Bessi, M. Coletto, G. A. Davidescu, A. Scala, G. Caldarelli, and W. Quattrociocchi. Science vs conspiracy: Collective narratives in the age of misinformation. PloS one, 10, 2015. doi: 10.1371 journal.pone.0118093pmid:25706981.

8. O. Oh, K. H. Kwon, and H. R. Rao. An exploration of social media in extreme events: Rumor theory and Twitter during the Haiti earthquake 2010. In Proceedings of the International Conference on Information Systems, page paper 231, 2010. doi: 10.1038/srep02980pmid: 24135961.

9. Rory Cellan-Jones. Who will pay for trusted news?, 2019.

10. Nic Newman, Richard Fletcher, Antonis Kalogeropoulos, and Rasmus Kleis Nielsen. Reuters Institute Digital News Report 2019. Technical report, University of Oxford, 2019.

11. Sepideh Bazazi, Jorina von Zimmermann, Bahador Bahrami, and Daniel Richardson. Selfserving incentives impair collective decisions by increasing conformity. PLOS ONE, 14(11): e0224725, 11 2019. ISSN 1932-6203. doi: 10.1371/journal.pone.0224725.

12. Albert B. Kao, Noam Miller, Colin Torney, Andrew Hartnett, and lain D. Couzin. Collective Learning and Optimal Consensus Decisions in Social Animal Groups. PLoS Computational Biology, 10(8):e1003762, 8 2014. ISSN 1553-7358. doi: 10.1371/journal.pcbi.1003762.

13. Marquis de Condorcet. Essai sur l'application de l'analyse à la probabilité des decisions rendues à la pluralité des vois. de l'Imprimerie Royale, Paris, 1785.

14. James Surowiecki. The Wisdom of Crowds. Why the Many are Smarter than the Few. Little, Brown Book Group, London, abacus edition, 2004.

15. Francis Galton. Vox Populi. Nature, 75(1949):450-451, 3 1907. ISSN 0028-0836. doi: 10.1038/075450a0.

16. James AR Marshall, Ralf HJM Kurvers, Jens Krause, and Max Wolf. Quorums enable optimal pooling of independent judgements in biological systems. eLife, 8, 2 2019. ISSN 2050-084X. doi: 10.7554/eLife.40368.

17. Krishna K. Ladha. The Condorcet Jury Theorem, Free Speech, and Correlated Votes. American Journal of Political Science, 36(3):617, 8 1992. ISSN 00925853. doi: 10.2307/ 2111584.

18. Joshua D. Angrist, Guido W. Imbens, and Donald B. Rubin. Identification of Causal Effects Using Instrumental Variables. Journal of the American Statistical Association, 91(434):444, 6 1996. ISSN 01621459. doi: 10.2307/2291629.

19. M. Lichman. UCI Machine Learning Repository [http://archive.ics.uci.edu/ml]., 2013.

20. R. S. Sutton and A. G. Barto. Reinforcement Learning: an introduction. MIT Press, Cambridge, MA, 1998.

21. Timothy E J Behrens, Laurence T Hunt, Mark W Woolrich, and Matthew F S Rushworth. Associative learning of social value. Nature, 456(7219):245-9, 11 2008. ISSN 1476-4687. doi: 10.1038/nature07538.

22. B. R. Newell and C. Schulze. Probability matching. In Cognitive Illusions: Intriguing Phenomena in Judgement, Thinking and Memory, chapter 3, page 504. Psychology Press, Abingdon, 2016.

23. Eric Schulz, Emmanouil Konstantinidis, and Maarten Speekenbrink. Explorationexploitation in a contextual multi-armed bandit task. In Proceedings of the 13th International Conference on Cognitive Modeling., Groningen, NL, 2015.

24. Andrew Lo. The adaptive market hypothesis., 2013.

25. Jan Lorenz, Heiko Rauhut, Frank Schweitzer, and Dirk Helbing. How social influence can undermine the wisdom of crowd effect. Proceedings of the National Academy of Sciences of the United States of America, 108(22):9020-5, 5 2011. ISSN 1091-6490. doi: 10.1073/ pnas. 1008636108.

26. Wataru Toyokawa, Andrew Whalen, and Kevin N. Laland. Social learning strategies regulate the wisdom and madness of interactive crowds. Nature Human Behaviour, 3(2):183-193, 2 2019. ISSN 2397-3374. doi: 10.1038/s41562-018-0518-x.

27. L. Rendell, R. Boyd, D. Cownden, M. Enquist, K. Eriksson, M. W. Feldman, L. Fogarty, S. Ghirlanda, T. Lillicrap, and K. N. Laland. Why Copy Others? Insights from the Socia Learning Strategies Tournament. Science, 328(5975):208-213, 4 2010. ISSN 0036-8075. doi: $10.1126 /$ science. 1184719 .

28. Rachel L. Kendal, Neeltje J. Boogert, Luke Rendell, Kevin N. Laland, Mike Webster, and Patricia L. Jones. Social Learning Strategies: Bridge-Building between Fields. Trends in Cognitive Sciences, 22(7):651-665, 7 2018. ISSN 13646613. doi: 10.1016/j.tics.2018.04. 003 .

29. Abdullah Almaatouq, Joshua Becker, James P. Houghton, Nicolas Paton, Duncan J. Watts, and Mark E. Whiting. Empirica: a virtual lab for high-throughput macro-level experiments. 62020.

\section{Bibliography}

1. Soroush Vosoughi, Deb Roy, and Sinan Aral. The spread of true and false news online. Science, 359(6380):1146-1151, 3 2018. ISSN 0036-8075. doi: 10.1126/science.aap9559.

2. David M. J. Lazer, Matthew A. Baum, Yochai Benkler, Adam J. Berinsky, Kelly M. Greenhill, Filippo Menczer, Miriam J. Metzger, Brendan Nyhan, Gordon Pennycook, David Rothschild, Michael Schudson, Steven A. Sloman, Cass R. Sunstein, Emily A. Thorson, Duncan J. Watts, and Jonathan L. Zittrain. The science of fake news. Science, 359(6380):1094-1096, 3 2018. ISSN 0036-8075. doi: 10.1126/science.aao2998.

3. Q. Mei Z. Zhao P. Resnick. Enquiring minds: Early detection of rumors in social media from enquiry posts. In Proceedings of the 24th International Conference on World Wide Web (ACM), page 1395-1405, 2015.

4. G. L. Ciampaglia, P. Shiralkar, L. M. Rocha, J. Bollen, F. Menczer, and A. Flammini. Computational fact checking from knowledge networks. PLOS ONE, 10(e0128193), 2015. doi: 10.1371/journal.pone.0128193pmid:26083336. 